\title{
Evaluation of Sclerotinia Stem Rot Resistance in Oilseed Brassica napus Using a Petiole Inoculation Technique Under Greenhouse Conditions
}

J. Zhao, National Key Laboratory of Crop Genetic Improvement, Huazhong Agricultural University, Wuhan, 430070, China, and Department of Agronomy, University of Wisconsin, Madison 53706; A. J. Peltier, Department of Plant Pathology, University of Wisconsin, Madison; J. Meng, National Key Laboratory of Crop Genetic Improvement, Huazhong Agricultural University; and T. C. Osborn, Department of Agronomy, and C. R. Grau, Department of Plant Pathology, University of Wisconsin, Madison

\begin{abstract}
Zhao, J., Peltier, A. J., Meng, J., Osborn, T. C., and Grau, C. R. 2004. Evaluation of Sclerotinia stem rot resistance in oilseed Brassica napus using a petiole inoculation technique under greenhouse conditions. Plant Dis. 88:1033-1039.

A petiole inoculation technique was adapted for evaluating resistance of oilseed Brassica napus seedlings to Sclerotinia sclerotiorum. In the first of four experiments, four isolates of S. sclerotiorum were tested, two originating from soybean and two from B. napus. In all, 10 to $47 \mathrm{~B}$. napus accessions were inoculated in the seedling stage and responses to isolates were evaluated using days to wilt (DW) and a lesion phenotype index (LP). There were no significant differences in virulence among the four isolates for DW and only slight differences for LP. However, significant differences $(P<0.0001)$ were observed among the $B$. napus accessions for DW and LP in this experiment and in subsequent experiments using one isolate. The responses of accessions were consistent among experiments and among evaluation criteria. Higher levels of resistance were found among winter-type than spring-type accessions, and among rapeseed-quality compared with canola-quality accessions. The most resistant accessions identified also were the most resistant when inoculated at the flowering stage. Terminal stems were inoculated immediately below the lowest flower and stem lesion length (SLL) was used to characterize the interaction phenotype of each accession. The petiole inoculation technique can be used successfully to differentiate oilseed B. napus germ plasm for response to $S$. sclerotiorum. This inoculation technique and the sources of resistance identified in this study may be used to determine inheritance resistance to $S$. sclerotiorum and for improving oilseed $B$. napus cultivars for resistance to this important pathogen.
\end{abstract}

Additional keywords: white mold

Sclerotinia sclerotiorum has been reported to infect more than 400 plant species, including several agronomically important crops, such as soybean (Glycine max), sunflower (Helianthus annuus), alfalfa (Medicago sativa), and oilseed (Brassica napus) $(6,28)$. This ascomycete fungus produces oxalic acid, which creates an optimal $\mathrm{pH}$ for the activity of enzymes related to pathogenesis, including cellwall-degrading enzymes $(8,9,20)$. Oxalic acid also has a general toxic effect on host tissues, which is believed to be responsible in part for the wide host range of S. sclerotiorum (6).

B. napus, traditionally cultivated in Canada, Europe, Australia, China, and more recently in the United States, is an

Corresponding author: C. R. Grau

E-mail: cg6@plantpath.wisc.edu

This work was funded by the United States Department of Agriculture Sclerotinia Initiative.

Accepted for publication 21 May 2004.

Publication no. D-2004-0714-01R

(c) 2004 The American Phytopathological Society important oilseed crop and contributes $10 \%$ of the world's production of edible oils (25). Seed quality has been improved by plant breeding, and cultivars with canola quality (low glucosinolate and low erucic acid content) have been released and are cultivated worldwide. Sclerotinia stem rot, often called white mold, is one of the most serious disease problems in canola-growing areas in the world and causes significant seed yield loss $(25,34)$. Fungicides have been used, but their efficacy is dependent on timing applications to match plant development and environmental conditions that favor infection (34). Furthermore, due to the economic and environmental concerns associated with fungicides, selection of cultivars resistant to S. sclerotiorum is a major priority for canola breeding.

Resistance to $S$. sclerotiorum has been evaluated in segregating populations and reported to be under monogenic or polygenic control in different plant species $(2,4)$. The responses of various hosts to $S$. sclerotiorum are highly dependent upon the environment in which they interact $(1,26,29,32)$, which complicates phenotypic classification of host genotypes. Molecular markers have been used to map genes that confer resistance to $S$. sclerotiorum in common (18) and dry bean (Phaseolus vulgaris) (21), sunflower (5), soybean (3), and B. napus (37), resulting in the identification and mapping of several potential quantitative trait loci (QTL) for resistance. Commonly encountered problems in these studies are that individual QTL effects are small and highly influenced by environment. Kolkman and Kelly (17) reported that higher levels of resistance in common bean were associated with lower canopy height and less lodging. Thus, relationships between agronomic traits and physiological resistance to $S$. sclerotiorum may increase the difficulty of selecting lines with resistance that is stable across diverse environments. The lack of highly resistant accessions within plant species has hampered the identification, characterization, and subsequent application of novel genes that confer resistance to $S$. sclerotiorum.

Several methods have been used to identify resistance to $S$. sclerotiorum in soybean, sunflower, and common bean $(10,13,15,16,24,35,36)$, including a detached leaf inoculation, an oxalate resistance test, and stem inoculation or sclerotia-seeded semi-natural field evaluations. It is common for responses of cultivars to vary among methods and experiments (36). Kim et al. (14) reported that ratings for soybean cultivars with intermediate reactions to $S$. sclerotiorum were not consistent across tests. Field tests of $S$. sclerotiorum resistance in B. napus have been reported, but no sources of complete resistance have been identified (38). Tolerance to oxalic acid was used to test the resistance of transgenic B. napus, but the results suggested that further evaluations were needed to refine this method (33). Sun (31) reported consistent results for widely divergent resistant and susceptible spring-type canola accessions using several inoculation methods, but results for accessions with intermediate ratings varied among methods. Thus, a more reliable method is needed to characterize $B$. napus accessions for reaction to $S$. sclerotiorum.

The goal of this study was to adapt a petiole inoculation technique (PIT), developed previously to challenge soybean accessions with $S$. sclerotiorum (12), to $B$. napus. This method has been used success- 
fully to screen soybean germ plasm and has gained acceptance due to its simplicity, consistency, and inexpensive nature. Objectives were to (i) characterize the virulence of $S$. sclerotiorum isolates, isolated from soybean and canola, to accessions of B. napus, and (ii) to characterize several widely investigated $B$. napus accessions for response to $S$. sclerotiorum to determine their potential for use in genetic studies and B. napus improvement.

\section{MATERIALS AND METHODS}

Plant material and growing conditions. A diverse range of B. napus germ

Table 1. Growth habit, seed quality, and origin of 47 Brassica napus accessions used in this study

\begin{tabular}{|c|c|c|}
\hline $\begin{array}{l}\text { Growth habit, } \\
\text { accession }\end{array}$ & Origin & $\begin{array}{c}\text { Seed } \\
\text { quality }^{\mathrm{a}}\end{array}$ \\
\hline \multicolumn{3}{|l|}{ Spring type } \\
\hline Marnoo & Australia & LL \\
\hline Westar & Canada & LL \\
\hline Nugget & Canada & $\mathrm{HH}$ \\
\hline Oro & Canada & LH \\
\hline Turret & Canada & $\mathrm{HH}$ \\
\hline Stellar & Canada & LL \\
\hline Quantum & Canada & LL \\
\hline Regent & Canada & LL \\
\hline Tower & Canada & LL \\
\hline Midas & Canada & LH \\
\hline P1804 & Canada & LL \\
\hline 44A89 & Canada & LL \\
\hline $44 \mathrm{~A} 76$ & Canada & LL \\
\hline Romeo & France & $\mathrm{HH}$ \\
\hline Furax & Germany & $\mathrm{HH}$ \\
\hline Kosa & Germany & LH \\
\hline Mazowiecki & Poland & $\mathrm{HH}$ \\
\hline Bronowski & Poland & LL \\
\hline Crusher & Sweden & LL \\
\hline Topas & Sweden & LL \\
\hline Nilla:1022 & Sweden & $\mathrm{HH}$ \\
\hline RV128 & United States & LL \\
\hline \multicolumn{3}{|l|}{ Winter type } \\
\hline Winfield & Canada & $\mathrm{HH}$ \\
\hline CK821 & China & LL \\
\hline NingRS-1 & China & $\mathrm{HH}$ \\
\hline H5200 & China & LL \\
\hline RV289 & China & LL \\
\hline 888 & China & LL \\
\hline Ning you 7 & China & $\mathrm{HH}$ \\
\hline Gan you 4 & China & $\mathrm{HH}$ \\
\hline Jet Neuf & Denmark & LH \\
\hline Liropa & Denmark & LL \\
\hline Major & France & $\mathrm{HH}$ \\
\hline Samourai & France & LL \\
\hline Tapidor & France & LL \\
\hline Rafal & France & LH \\
\hline Barcoli & Germany & $\mathrm{HH}$ \\
\hline Santana & Germany & LL \\
\hline Barsica & Germany & $\mathrm{HH}$ \\
\hline Expander & Germany & LH \\
\hline Elena & Germany & LL \\
\hline Norin8 & Japan & $\mathrm{HH}$ \\
\hline Norin39 & Japan & $\mathrm{HH}$ \\
\hline Mar & Poland & LL \\
\hline Warszawski & Poland & $\mathrm{HH}$ \\
\hline Bolko & Poland & LL \\
\hline Marcus & Netherlands & $\mathrm{HH}$ \\
\hline
\end{tabular}

${ }^{\mathrm{a}} \mathrm{LL}=$ low erucic acid and low glucosinolate content, $\mathrm{HH}=$ high erucic acid and high glucosinolate content, and $\mathrm{LH}=$ low erucic acid and high glucosinolate content. plasm was used in this study, including accessions with different growth habits and seed qualities (Table 1). H5200 was kindly provided by $\mathrm{T}$. $\mathrm{Fu}$, Huazhong Agricultural University, China. NingRS-1, 888, Ning you 7, and CK821 were from stocks held by J. Meng, Huazhong Agricultural University, China. RV289 is a single plant selected from Hua dbl2. P1804 is a doubled haploid (DH) line having the malesterility restorer gene of the SeedLink transgenic system (19). Major, Stellar, Quantum, and Crusher are accessions acquired by T. Osborn, University of Wisconsin-Madison, from various sources. All other accessions were provided by the United States Department of Agriculture (USDA) North Central Regional Plant Introduction Station, Iowa State University.

Seed were planted in Scott's Metro-mix 336p (Scott's-Sierra Horticultural Products Co., Marysville, OH) into 8-cm-diameter (experiments 1 to 3 ) or $12-\mathrm{cm}$-diameter (experiment 4) pots. Plants were thinned to one plant per pot and were grown in a greenhouse under 350 to $500 \mu \mathrm{mol} \mathrm{m} \mathrm{m}^{-2} \mathrm{~s}^{-1}$ lights (16-h photoperiod). For experiments 1 and 2 , temperatures were set at $24^{\circ} \mathrm{C}$ during the day and 19 to $20^{\circ} \mathrm{C}$ at night; however, during experiment 1 , the temperature reached 26 to $27^{\circ} \mathrm{C}$ during some days due to hot weather outside. The temperature for experiments 3 and 4 was set at a constant $21^{\circ} \mathrm{C}$. Experiments 1, 2, and 4 included 10 accessions and experiment 3 had 47 accessions. Vernalization treatments $\left(4^{\circ} \mathrm{C}\right)$ of 2,4 , and 9 weeks were applied to spring accessions, Chinese winter accessions, and cv. Major, respectively.
Accessions were planted on different dates, such that all plants were moved from vernalization chambers to the greenhouse on the same day. Using this design, all plants received some cold treatment. Most plants of accessions of CK821, NingRS-1, H5200, RV289, 888, and Crusher flowered within a few days of each other and were inoculated on the same day. Plants of accessions of Stellar, Quantum, and P1804 flowered a little later but within a few days of each other and were inoculated 3 days later. Plants of Major, which flowered considerably later, were dropped from the experiment.

Pathogen isolates and growing conditions. Four isolates of S. sclerotiorum, designated Ramsey, Benson, 105HT, and Fond du Lac, were used in experiment 1. Isolates Ramsey and Benson originated from B. napus in 2002 and originated in correspondingly named counties in North Dakota, and were provided by Carl Bradley and Luis Del Rio, North Dakota State University. The Fond du Lac isolate, collected in 2000, originated from infected soybeans in Fond du Lac County, Wisconsin. Isolate $105 \mathrm{HT}$ was isolated from soybean seed produced near Story City, IA in 1996 and was provided by Glen Hartman, USDA-Agricultural Research Services, Urbana, IL. Isolate $105 \mathrm{HT}$ was used in subsequent experiments because it had been used in previous research on $S$. sclerotiorum, it expressed a high level of virulence, and it has remained stable for virulence after repeated culturing (35). Prior to each experiment, isolates were cultured on potato dextrose agar (PDA; 25\% potato

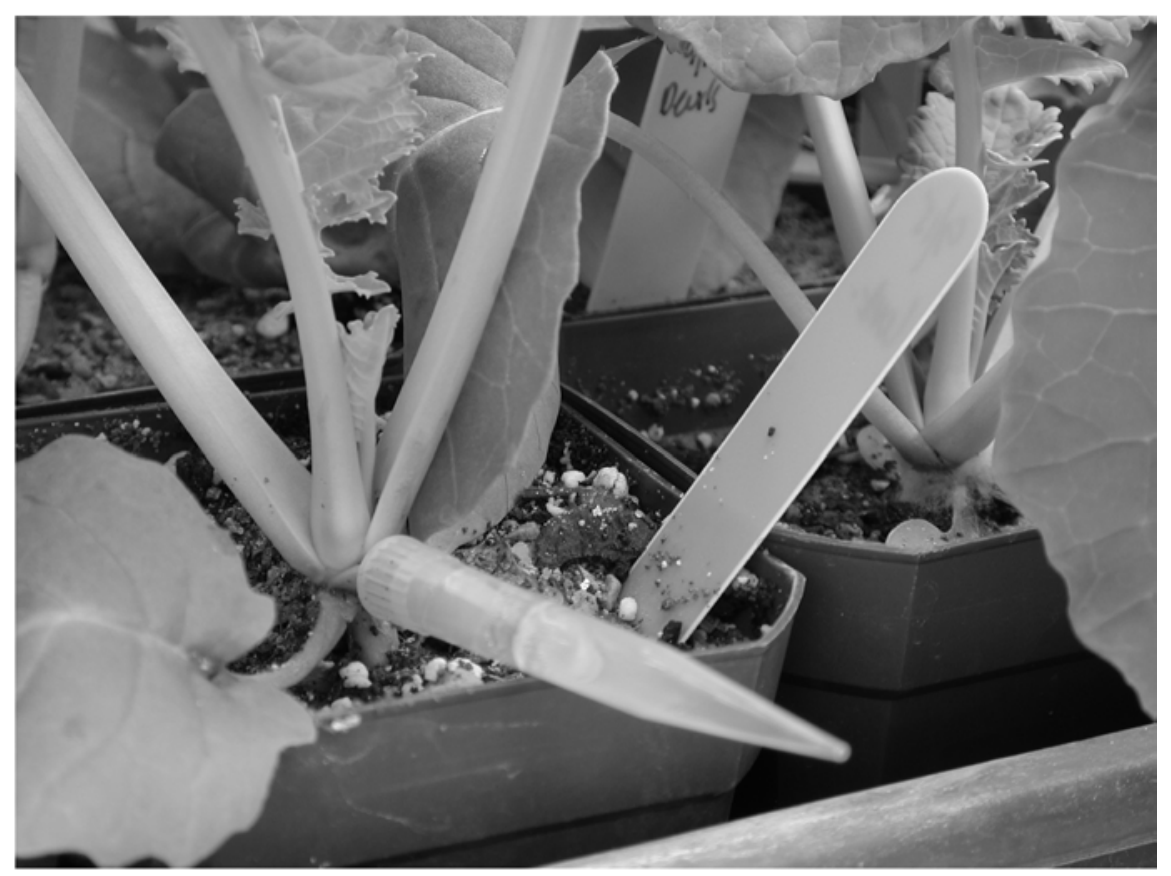

Fig. 1. Petioles of the third fully expanded leaf were severed $2.5 \mathrm{~cm}$ from the main stem using a razor blade. The tapered end of a 1,000- $\mu$ pipette tip filled with potato dextrose agar and Sclerotinia sclerotiorum mycelium was plugged with an index finger, and the petiole was forced through the agar plug into the pipette until the petiole tip lay flush with the agar plug. All pipette tips remained on inoculated petioles until the end of experiments or until petioles abscised. 
extract, $2 \%$ dextrose, $1.5 \%$ agar) and incubated at 22 to $25^{\circ} \mathrm{C}$ under $45 \mu \mathrm{mol} \mathrm{m} \mathrm{m}^{-2} \mathrm{~s}^{-1}$ wide-spectrum lights (12-h photoperiod).

Inoculation technique. The PIT was adapted from the method described by Hoffman et al. (12) for challenging soybean germ plasm with $S$. sclerotiorum. The open end of a 1,000- $\mu$ l pipette tip was pushed into the margin of a 3-day-old colony to acquire a 8-mm-thick plug of PDA and mycelium. Pipette tips were preloaded and transported in sealed pipette tip boxes prior to inoculation of plants.
Petioles of the third fully expanded leaf were selected as the infection court of 4week-old seedlings for experiments 1,2 , and 3. Petioles were severed $2.5 \mathrm{~cm}$ from the main stem using a razor blade. The tapered end of each inoculum-filled pipette tip was covered with an index finger, and the petiole was forced through the agar plug within the pipette tip until the petiole end lay flush with the end of the agar plug (Fig. 1). Plants in experiment 4 were inoculated when each plant had at least one open flower. Plants were 7 to 8 weeks old

Table 2. Responses of 10 Brassica napus accessions to Sclerotinia sclerotiorum after inoculation using a petiole inoculation technique ${ }^{\mathrm{a}}$

\begin{tabular}{|c|c|c|c|c|}
\hline \multirow[b]{2}{*}{ Accession } & \multicolumn{2}{|c|}{ Experiment $1^{b}$} & \multicolumn{2}{|c|}{ Experiment $2^{c}$} \\
\hline & DW & $\mathbf{L P}$ & DW & LP \\
\hline CK821 & 7.9 & $0.06(0.05,0.09)$ & 11.5 & $0.18(0.12,0.30)$ \\
\hline NingRS-1 & 6.3 & $0.33(0.22,0.46)$ & 11.9 & $0.19(0.14,0.26)$ \\
\hline Major & 6.5 & $0.33(0.26,0.41)$ & 11.3 & $0.39(0.29,0.50)$ \\
\hline RV289 & 5.1 & $0.49(0.38,0.59)$ & 11.1 & $0.29(0.20,0.41)$ \\
\hline H5200 & 5.7 & $0.35(0.26,0.46)$ & 7.2 & $0.60(0.47,0.72)$ \\
\hline 888 & 5.5 & $0.58(0.49,0.67)$ & 8.6 & $0.54(0.45,0.69)$ \\
\hline Quantum & 4.5 & $0.62(0.53,0.70)$ & 7.1 & $0.71(0.68,0.73)$ \\
\hline Crusher & 3.4 & $0.74(0.72,0.77)$ & 6.0 & $0.71(0.68,0.73)$ \\
\hline Stellar & 3.1 & $0.75(0.72,0.77)$ & 5.7 & $0.71(0.68,0.73)$ \\
\hline P1804 & 3.3 & $0.75(0.72,0.77)$ & 5.4 & $0.71(0.68,0.73)$ \\
\hline Trial mean & 5.1 & 0.50 & 8.6 & 0.50 \\
\hline $\operatorname{LSD}(0.05)^{\mathrm{d}}$ & 0.6 & NA & 1.4 & NA \\
\hline
\end{tabular}

${ }^{a}$ Days to wilt (DW) was determined by the number of days from inoculation to irreversible wilt of plants. Lesion phenotype (LP) was determined by a 0-to-4 category index used to score each challenged plant on the final day of days to wilt evaluations. $0=$ no symptom, no lesion, no water-soaking and no wilt; 1 = small lesion at junction of petiole and stem, no water-soaking, and no wilt; 2 = small water-soaked lesion and no wilt; 3 = expanded, sunken water-soaked lesion and no wilt; and 4 = expanded, sunken, water-soaked lesion resulting in irreversible wilt of foliage. Data represent the estimated relative treatment effects calculated by the method of Shah and Madden (2004). Confidence interval (95\%) for relative treatment effects are presented in parentheses. Values of estimated relative treatments larger than 0.50 in experiments 1 and 2 illustrate that these treatments tended to have larger values compared with the observations from all treatments combined.

${ }^{b}$ Mean responses (DW) and estimated relative treatment effects (LP) of accessions (15 replicate plants) inoculated with four isolates. Results represent the combined data for the four isolates tested.

${ }^{c}$ Mean responses (DW) and estimated relative treatment effects (LP) of accessions (14 replicate plants) inoculated with isolate 105HT.

${ }^{\mathrm{d}}$ LSD $=$ least significant difference for comparing individual accessions at $P=0.05$; NA $=$ not applicable. at inoculation. The top part of the main stem was severed with a razor blade at the internode below the first open flower. The cut end of the main stem was inoculated as described for petioles.

Response scoring. Two separate scoring systems were used to classify phenotypic responses for each challenged plant in experiments 1,2 , and 3 . The response of individual plants of each accession was determined by days to wilt (DW). Wilted plants were recorded daily beginning the first day following inoculation. All DW values corresponded to the day that a plant exhibited an irreversible wilt phenotype. A plant was deemed wilted when tissues of the apical meristem were limp or the leaves were flaccid. Plants were assessed each day for 7,11 , and 10 consecutive days for experiments 1,2 , and 3 , respectively. This variation in days of data collection was required because higher temperatures during experiment 1 caused accelerated disease development and wilt of most plants by day 7. Plants that had not wilted by day 7 were given a score of 8 in experiment 1 , plants not wilted by day 11 were given a score of 12 in experiment 2 , and a score of 11 was assigned to plants not wilted by day 10 in experiment 3 . A lesion phenotype (LP) index was devised in order to classify phenotypes for all inoculated plants. The LP index consisted of a 0-to-4 scale and was assessed on the final day of wilt scoring. Lesion phenotypes were categorized as follows: $0=$ no symptom, no lesion, no water-soaking, and no wilt; 1 = small lesion at junction of petiole and stem, no water-soaking, and no wilt; 2 $=$ small water-soaked lesion and no wilt; 3 = expanded, sunken water-soaked lesion and no wilt; and 4 = expanded, sunken, water-soaked lesion resulting in irreversible wilt of foliage. Stem lesion length (SLL; cm) was measured 4, 7, and 10 days

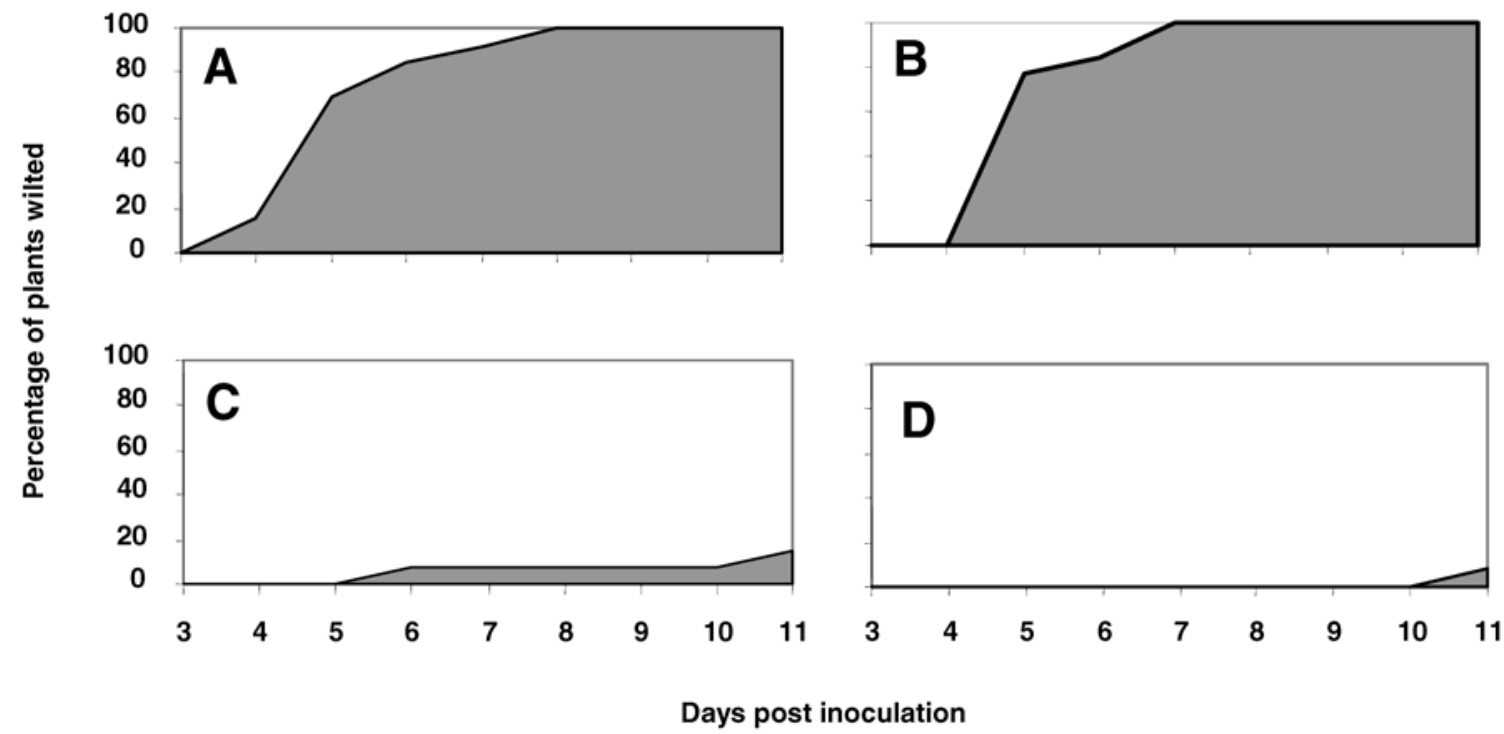

Fig. 2. Disease progress curves for two susceptible (Stellar and P1804) and two partially resistant (CK821 and NingRS-1) Brassica napus accessions challenged in experiment 2 with Sclerotinia sclerotiorum using a petiole inoculation technique. A, Stellar, B, P1804, C, CK821, and D, NingRS-1. 
Table 3. Reponses of 47 Brassica napus accessions to Sclerotinia sclerotiorum after inoculation using a petiole inoculation technique

\begin{tabular}{|c|c|c|}
\hline Accession & DW $^{\mathbf{a}}$ & $\mathbf{L P} \mathbf{P}^{\mathbf{b}}$ \\
\hline Marnoo & 5.4 & $0.55(0.54,0.56)$ \\
\hline Westar & 4.2 & $0.55(0.54,0.56)$ \\
\hline Nugget & 5.8 & $0.55(0.54,0.56)$ \\
\hline Oro & 5.1 & $0.55(0.54,0.56)$ \\
\hline Turret & 5.0 & $0.55(0.54,0.56)$ \\
\hline Stellar & 4.4 & $0.55(0.54,0.56)$ \\
\hline Quantum & 6.1 & $0.55(0.54,0.56)$ \\
\hline Regent & 5.1 & $0.55(0.54,0.56)$ \\
\hline Tower & 4.5 & $0.55(0.54,0.56)$ \\
\hline Midas & 4.8 & $0.55(0.54,0.56)$ \\
\hline P1804 & 5.3 & $0.55(0.54,0.56)$ \\
\hline $44 \mathrm{~A} 89$ & 5.4 & $0.55(0.54,0.56)$ \\
\hline $44 \mathrm{~A} 76$ & 4.8 & $0.55(0.54,0.56)$ \\
\hline Romeo & 6.6 & $0.51(0.43,0.59)$ \\
\hline Furax & 6.1 & $0.55(0.54,0.56)$ \\
\hline Kosa & 5.2 & $0.55(0.54,0.56)$ \\
\hline Mazowiecki & 5.5 & $0.51(0.44,0.58)$ \\
\hline Bronowski & 5.0 & $0.55(0.54,0.56)$ \\
\hline Crusher & 5.1 & $0.55(0.54,0.56$ \\
\hline Topas & 6.1 & $0.55(0.54,0.56)$ \\
\hline Nilla:1022 & 7.6 & $0.48(0.39,0.57)$ \\
\hline RV128 & 5.4 & $0.55(0.54,0.56)$ \\
\hline Marcus & 6.5 & $0.52(0.45,0.58)$ \\
\hline Bolko & 6.7 & $0.52(0.45,0.58)$ \\
\hline Winfield & 6.4 & $0.55(0.54,0.56)$ \\
\hline CK821 & 10.3 & $0.11(0.04,0.26)$ \\
\hline NingRS-1 & 8.4 & $0.29(0.17,0.44)$ \\
\hline H5200 & 7.2 & $0.48(0.38,0.57)$ \\
\hline RV289 & 7.2 & $0.48(0.39,0.57)$ \\
\hline 888 & 6.1 & $0.55(0.54,0.56)$ \\
\hline Ning you 7 & 8.4 & $0.45(0.35,0.55)$ \\
\hline Gan you 4 & 9.1 & $0.30(0.19,0.45)$ \\
\hline Jet Neuf & 7.4 & $0.48(0.39,0.57)$ \\
\hline Liropa & 7.6 & $0.48(0.38,0.57)$ \\
\hline Major & 8.1 & $0.41(0.31,0.53)$ \\
\hline Samourai & 5.6 & $0.55(0.54,0.56)$ \\
\hline Tapidor & 5.3 & $0.55(0.54,0.56)$ \\
\hline Rafal & 7.2 & $0.48(0.39,0.57)$ \\
\hline Barcoli & 6.9 & $0.55(0.54,0.56)$ \\
\hline Santana & 5.8 & $0.55(0.54,0.56)$ \\
\hline Barsica & 6.9 & $0.55(0.54,0.56)$ \\
\hline Expander & 8.9 & $0.33(0.21,0.48)$ \\
\hline Elena & 6.0 & $0.52(0.45,0.58)$ \\
\hline Norin8 & 7.5 & $0.43(0.32,0.55)$ \\
\hline Norin39 & 6.8 & $0.41(0.28,0.55)$ \\
\hline Mar & 5.2 & $0.55(0.54,0.56)$ \\
\hline Warszawski & 6.2 & $0.48(0.38,0.57)$ \\
\hline $\operatorname{LSD}(0.05)^{\mathrm{c}}$ & 1.3 & NA \\
\hline
\end{tabular}

${ }^{a}$ Days to wilt (DW), determined by the number of days from inoculation to irreversible wilt of plants. Values reported are means based on 14 replications.

${ }^{\mathrm{b}}$ Lesion phenotype (LP), determined by a 0-to-4 category index scored to each challenged plant on the final day of days to wilt evaluations. $0=$ no symptoms at node nor wilt; $1=$ small lesion at junction of petiole and stem, no water-soaking, and no wilt; $2=$ small water-soaked lesion and no wilt; 3 = expanded, sunken water-soaked lesion and no wilt; and 4 = expanded, sunken, watersoaked lesion resulting in irreversible wilt of foliage. Data represent the estimated relative treatment effects calculated by the method of Shah and Madden (2004) based on 14 replications. Confidence interval (95\%) for relative treatment effects are presented in parentheses. Values larger than 0.50 illustrate that these treatments tended to have larger values compared with the observations from all treatments combined.

${ }^{c}$ LSD $=$ least significant difference for comparing individual accessions at $P=0.05$. NA $=$ not applicable. after inoculation in experiment 4 . We did not measure DW or LP in experiment 4 because the meristem, which is most sensitive to wilt, had been removed, and the LP was very different for the plants inoculated on the main stem.

Experimental design and data analysis. Experiment 1 involved four isolates and 10 host accessions arranged in a splitplot experimental design with accessions as whole plots, isolates as subplots, and 15 replications. Experiments 2 and 3, in which only isolate $105 \mathrm{HT}$ was used, included 10 and 47 accessions, respectively, and were conducted as randomized complete block designs (RCBD) with 14 replications. Experiment 4 included the same accessions as experiment 2, but with 10 replications.

The DW and SLL data for the experiments were analyzed by the PROC Mixed procedure in SAS (2001, version 8.2). Accessions were treated as whole-plot factors and isolates as subplot factors in the split-plot design of experiment 1 . In subsequent experiments, accessions were analyzed as fixed effects and blocks as random effects. Means were compared by least significant differences at $P=0.05$.

The LP data was analyzed by analysis of variance (ANOVA)-type statistics (ATS) (30). Data first were ranked, and then PROC Mixed procedure and macros (30) in SAS (2001, version 8.2) were used to calculate the estimated relative treatment effects and corresponding variances. No isolate accession interaction was detected; therefore, measurements from four isolates were averaged in experiment 1 and blocks were treated as replications. Pearson's correlation coefficients of DW were calculated using the PROC CORR procedure in SAS (2001, version 8.2) to compare mean scores between experiments. Spearman's rho was calculated to compare estimated relative treatment effects between experiments. The summary results of DW and LP from each experiment were correlated with Spearman's rho in SAS (2001, version 8.2).

\section{RESULTS}

There were no significant differences in DW among the four isolates of S. sclerotiorum used to challenge accessions of B. napus in experiment 1 and only slight difference between two isolates (Benson and 105HT) for LP (data not shown). Isolate-accession interactions were not observed $(P=0.44$ for DW and $P=0.15$ for LP). Thus, results from all four isolates in experiment 1 were averaged for each accession (Table 2), and a single isolate $(105 \mathrm{HT})$ was used as inoculum for experiments 2,3 , and 4 .

In experiment 1 , plants began to wilt at day 3 after inoculation and scoring ended by day 7 . The 10 accessions were significantly different $(P<0.0001)$ for mean DW based on an ANOVA treatment of the data, and for the distribution of LP scores based on ATS analysis (Table 2). Stellar, Crusher, and P1804 were the most susceptible and most plants of these accessions had died by day 4 . CK821 was the most resistant accession and had a DW score close to 8 . LP scores were highly correlated with DW scores $\left(\rho_{\text {DW } 1, \mathrm{LP} 1}=0.96, P<0.0001\right)$.

In experiment 2 , plants developed symptoms more slowly than in experiment 1 , probably due to higher ambient air temperature. However, the DW and LP scores were highly correlated between the two experiments $\left(r_{\mathrm{DW} 1, \mathrm{DW} 2}=0.89, P<0.0004\right.$; $\left.\rho_{\mathrm{LP} 1, \mathrm{LP} 2}=0.92, P<0.0002\right)$. The 10 accessions were significantly different $(P<$ $0.0001)$ for DW based on ANOVA and for the distribution of LP scores based on ATS (Table 2). Stellar, Crusher, and P1804 again were the most susceptible, and most plants of these accessions had died by day 6. CK821, NingRS-1, Major, and RV289 were the most resistant and had DW scores close to 12 . The LP scores were highly correlated with DW scores $\left(\rho_{\text {DW2,LP2 }}=\right.$ 0.94, $P<0.0001)$.

The responses of two resistant and two susceptible accessions used in experiment 2 were compared by disease progress curves (Fig. 2). The highly susceptible accessions Stellar and P1804 began to wilt by day 3 or 4 and close to $100 \%$ of plants wilted by day 7 (Fig. 2A and B). For the resistant accession CK821, only one plant wilted by day 5 , with just two wilted plants by the end of day 11 (Fig. 2C). Accession NingRS-1 had only one plant wilted by the last scoring date (Fig. 2D).

Forty-seven accessions with different growth habits and seed qualities were screened in experiment 3 . The accessions were significantly different $(P<0.0001)$ for DW based on ANOVA and for the distribution of LP based on ATS, and the DW and LP scores were highly correlated $\left(\rho_{\text {DW } 3, \mathrm{LP} 3}=0.82, P<0.0001\right.$; Table 3$)$. The most resistant (CK821, NingRS-1, Major, and RV289) and most susceptible (Stellar, P1804, Crusher, and Quantum) accessions identified in experiments 1 and 2 showed the same trends in experiment 3. Based on country of origin, the Chinese accessions were most resistant and the Canadian accessions were most susceptible (Fig. 3). Also, winter accessions tended to show more resistance than spring accessions, and noncanola-quality accessions exhibited more resistance than canola-quality accessions within each growth habit type (Fig. 4).

The same 10 accessions used in experiments 1 and 2 were inoculated at the flowering stage in experiment 4. Only nine accessions were included in the analysis because the European winter-type cv. Major flowered significantly later than the other accessions. The accessions did not differ for stem lesion length at day 4 , but several accessions exhibited longer lesions $(P<0.0001)$ by day 7 . By day 10 , lesion expansion on susceptible accessions was even greater compared with resistant ac- 
cessions (Table 4). The most resistant accessions (CK821, RV289, and NingRS-1), identified in previous experiments when the plants were inoculated at the seedling stage also showed high levels of resistance when inoculated at the flowering stage in experiment 4.

\section{DISCUSSION}

The adapted PIT from soybean was found to be an effective method to distinguish responses of $B$. napus accessions challenged by $S$. sclerotiorum. At the beginning of infection, white mycelia was visible on the surface of petioles. Petioles decayed to the point of attachment to the main stem in 2 to 3 days, depending on host accessions. Water-soaked lesions and symptoms of wilt were observed on day 3 after inoculation for the most susceptible accessions. Sclerotia were formed at later stages of disease development. For the most resistant accessions, the water-soaked lesion was restricted to the inoculated node and plants did not wilt. It is possible that plants could escape infection. However, experiments were repeated and interaction phenotypes were consisted for accessions characterized as susceptible and resistant. All plants of accessions characterized as susceptible always wilted by the completion of each experiment.
The four isolates of $S$. sclerotiorum compared in experiment 1 did not differ in overall virulence on B. napus, nor did they interact differentially with specific accessions. Pratt and Rowe (27) also reported that alfalfa accessions responded similarly to five isolates of $S$. sclerotiorum with no significant isolate-accession interactions. These results suggest that isolates of $S$. sclerotiorum have not developed specificity for canola accessions, which is consistent with the wide host range of $S$. sclerotiorum and the nonspecificity of hostpathogen interactions (6). Two phenotypic scoring criteria at the seedling stage, DW and an LP index, were used to identify

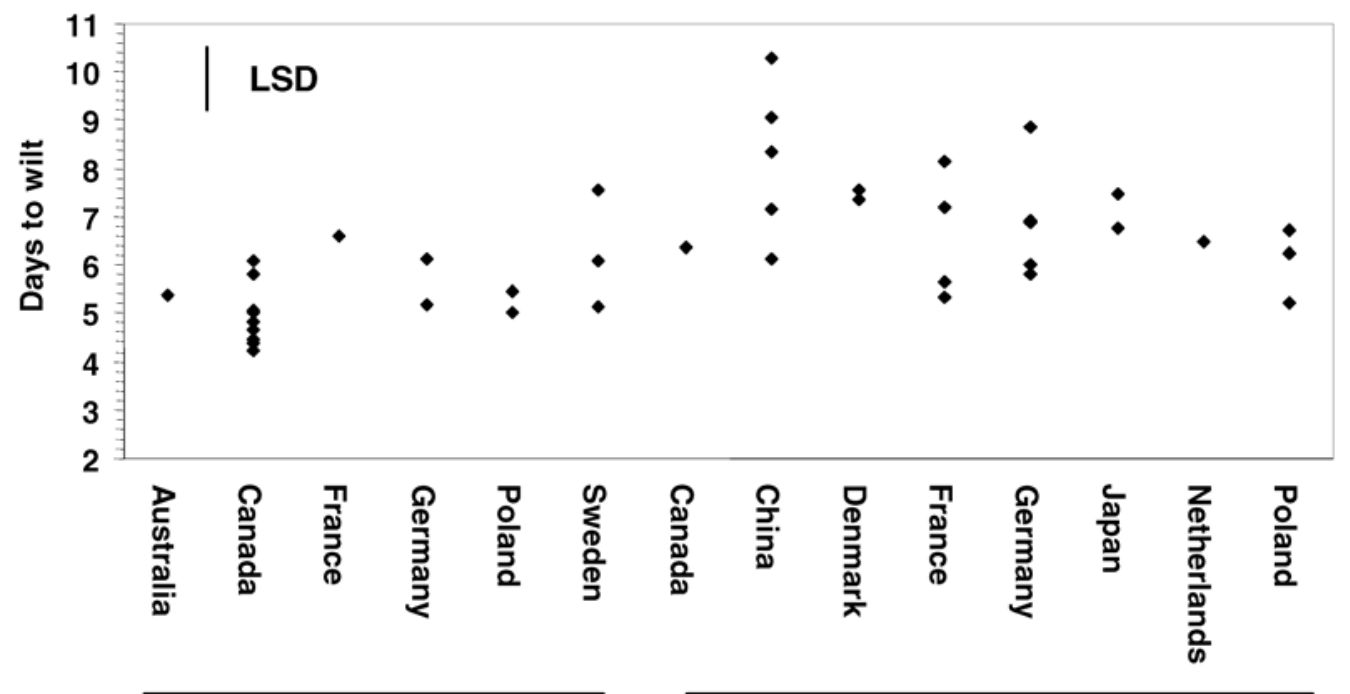

Spring-type

Winter-type

Fig. 3. Responses to Sclerotinia sclerotiorum (days to wilt) of Brassica napus accessions based on country of origin and growth habit. Least significant difference value shown for $P<0.05$.

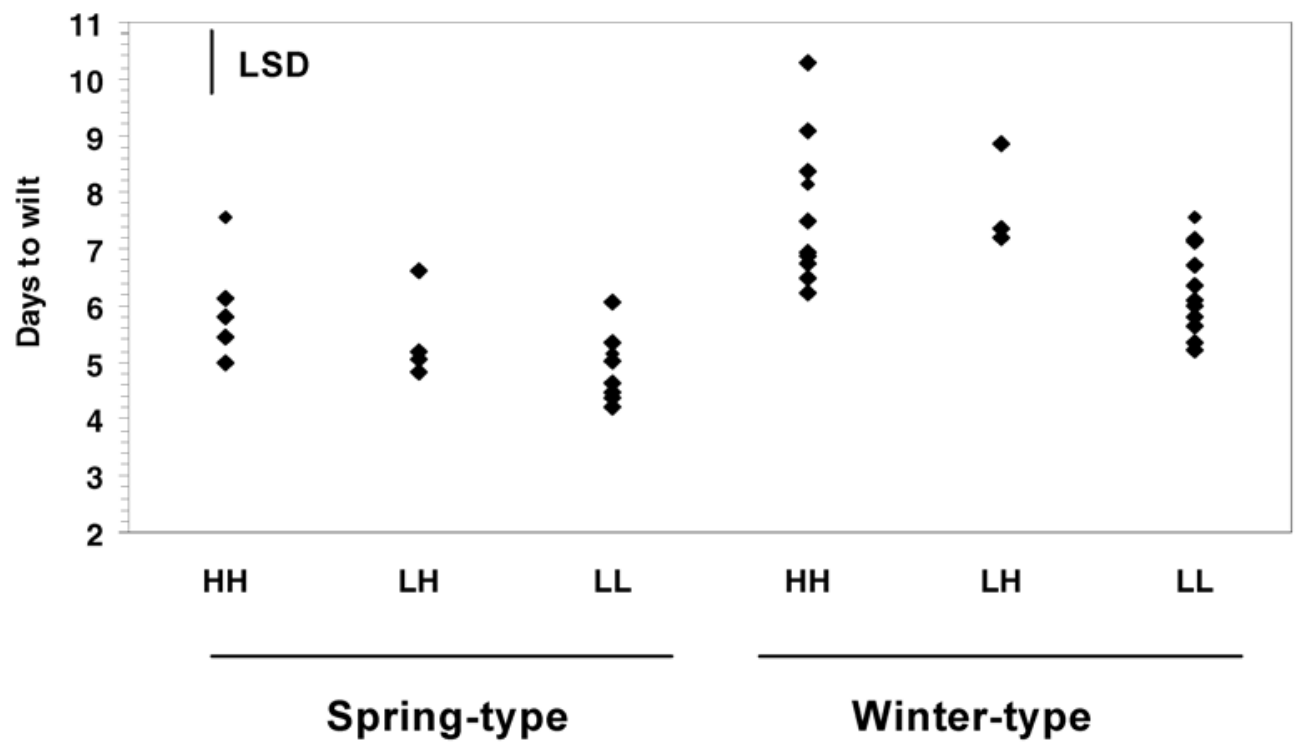

Fig. 4. Responses to Sclerotinia sclerotiorum (days to wilt) of Brassica napus accessions based on seed quality and growth habit. Least significant difference value shown for $P<0.05$. $\mathrm{HH}=$ high erucic acid and high glucosinolate content, $\mathrm{LH}=$ low erucic acid and high glucosinolate content, and $\mathrm{LL}=$ low erucic acid and low glucosinolate content. 
highly resistant and highly susceptible accessions. Although symptoms progressed differently in each experiment, the rankings of accessions based on DW and LP were very similar among experiments, and the two criteria were highly correlated for the accessions evaluated. These results suggest that either criterion could be used to evaluate accessions of canola for resistance to $S$. sclerotiorum. The LP score was used to classify plant phenotypes at a single point in time late in the incubation period. Thus, this approach would not distinguish differences in disease progress among accessions that may be predictive of field performance in natural epidemics. However, the LP index may be more practical for screening a large number of accessions because it involves a single evaluation at the end of an experiment.

Accessions with winter growth habits tended to exhibit more resistance than accessions with spring growth habits at the seedling stage. Growth habit could have influenced the disease response of accessions because, after 4 weeks of growth, the internodes of spring accessions begin to elongate, but winter accessions continue to have short internodes. The close proximity of nodes in winter accessions may have an anatomical effect that slowed the progress of the pathogen into internode tissues, allowing more time for plant-mounted defense mechanisms. However, in experiment 3 , which included a larger number of accessions, some winter accessions (Samourai, Tapidor, Santana, MAR, and Elena) had lower DW scores (greater symptom severity) than some spring accessions (Nilla:1022, Romeo, and Furax) (Table 3). Furthermore, results from stem inoculations of flowering plants in experiment 4 suggested that the resistance observed for most winter accessions at the seedling stage was not related to growth habit. In experiment 4 , stems of flowering plants had similar internode lengths and the most resistant accessions also ex-

Table 4. Responses of nine Brassica napus accessions to inoculation with Sclerotinia sclerotiorum on the main stem at the flowering stage $^{\mathrm{a}}$

\begin{tabular}{lccc}
\hline Accession & SLL(4) & SLL(7) & SLL(10) \\
\hline CK821 & 4.2 & 6.0 & 6.4 \\
NingRS-1 & 4.4 & 5.7 & 6.1 \\
RV289 & 4.0 & 5.0 & 5.3 \\
H5200 & 4.5 & 7.3 & 9.1 \\
888 & 5.0 & 7.2 & 8.8 \\
Quantum & 4.4 & 6.3 & 7.9 \\
Crusher & 4.4 & 6.1 & 7.3 \\
Stellar & 5.0 & 7.2 & 8.9 \\
P1804 & 4.5 & 6.6 & 8.6 \\
Trial mean & 4.5 & 6.4 & 7.6 \\
LSD $(0.05)^{\mathrm{b}}$ & 0.9 & 0.9 & 1.0 \\
\hline
\end{tabular}

${ }^{a}$ SLL $=$ stem lesion length $(\mathrm{cm})$; numbers in parentheses indicate days after inoculation when measurements were recorded.

${ }^{\mathrm{b}}$ LSD $=$ least significant difference for comparing individual accessions at $P=0.05$. pressed resistance at the seedling stage. One accession that exhibited resistance at the seedling stage, H5200, was highly susceptible at flowering, suggesting that growth habit or growth stage may affect the response of some accessions. Thus, the potential effect of growth habit or developmental stage on disease response requires further study.

We found evidence of a relationship between canola quality and response to $S$. sclerotiorum within each growth habit type. Non-canola-quality accessions tended to be more resistant than canolaquality accessions. This is similar to what was reported by Sun (31) for spring-type canola. Mithen et al. (23) previously reported that high levels of resistance to Leptosphaeria maculans were related to high levels of leaf alkenyl glucosinolate content. However, the glucosinolate levels in vegetative tissues do not correspond to levels found in seed (7), and low and high glucosinolate oilseed cultivars have been shown to possess similar concentrations of leaf glucosinolates (22). Thus, the apparent relationship between canola quality and resistance to $S$. sclerotiorum may be due to the elimination of resistance alleles linked to alleles for high erucic acid or glucosinolates during breeding of canola-quality cultivars.

Results from this study demonstrate that the PIT developed for soybean can be used successfully to distinguish the response of oilseed B. napus accessions to S. sclerotiorum at the seedling stage. Similar responses were obtained by inoculating stems at the flowering stage, suggesting that the PIT may be useful for predicting the response of accessions in natural epidemics, which normally occur during the flowering stage. However, further studies are needed to determine if the PIT can be used to predict responses of accessions in natural epidemics in the field. Using the PIT, we identified several partially resistant accessions from China that were identified as partially resistant in previous studies (NingRS-1 [36] and CK821 [11]). Additional accessions (Major and RV289) also were identified and expressed higher levels of resistance. These accessions may be useful for studying the genetic control of resistance to $S$. sclerotiorum, and for creating breeding populations to improve the resistance of $B$. napus cultivars.

\section{ACKNOWLEDGMENTS}

We thank T. Fu, C. Bradley, G. Hartman, and L. Del Rio for providing accessions and isolates; and M. Quijada and P. Quijada for their assistance with the statistical analysis.

\section{LITERATURE CITED}

1. Abawi, G. S., and Grogan, R. G. 1978. Epidemiology of diseases caused by Sclerotinia species. Phytopathology 69:899-903.

2. Abawi, G. S., Provuidenti, R., Crosier, D. C., and Hunter, J. E. 1978. Inheritance of resistance to white mold disease in Phaseolus coccineus. J. Hered. 69:200-202.
3. Arahana, V. S., Graef, G. L., Specht, J. E., Steadman, J. R., and Eskridge, K. M. 2001. Identification of QTLs for resistance to Sclerotinia sclerotiorum in soybean. Crop Sci. 41:180-188.

4. Baswana, K. S., Rastogi, K. B., and Sharma, P. P. 1991. Inheritance of stalk rot resistance in cauliflower (Brassica oleracea var. Botrytis L.). Euphytica 57:93-96.

5. Bert, P. F., Jouan, I., Tourvieille de Labrouhe, D., Serre, F., Nicolas, P., and Vear, F. 2002. Comparative genetic analysis of quantitative traits in sunflower (Helianthus annuus L.) 1. QTL involved in resistance to Sclerotinia sclerotiorum and Diaporthe helianthi. Theor. Appl. Genet. 105:985-993.

6. Boland, G. J., and Hall, R. 1994. Index of plant hosts of Sclerotinia sclerotiorum. Can. J. Plant Pathol. 16:93-108.

7. Fieldsend, J., and Milford, G. F. J. 1994 Changes in glucosinolates during crop development in single- and double-low genotypes of winter oilseed rape (Brassica napus): I: Production and distribution in vegetative tissues and developing pods during development and potential role in the recycling of sulphur within the crop. Ann. Appl. Biol. 124:531-542.

8. Fraissinet-Trachet, L., Reymond-Cotton, P., and Fevre, M. 1995. Characterization of a multigene family encoding an endogalacturonase in Sclerotinia sclerotiorum. Curr. Genet. 29:96-99.

9. Godoy, G., Steadman, J. R., Dickman, M. B., and Dam, R. 1990. Use of mutants to demonstrate the role of oxalic acid in pathogenicity of Sclerotinia sclerotiorum on Phaseolus vulgaris. Physiol. Mol. Plant Pathol. 37:179-191.

10. Hartman, G. L., Gardner, M. E., Hymowitz, T., and Naidoo, G. C. 2000. Evaluation of perennial Glycine species for resistance to soybean fungal pathogens that cause Sclerotinia stem rot and sudden death syndrome. Crop Sci. 40:545-549.

11. He, Y., and Chen, X. 1989. Cultivation and significance of a new multi-resistant variety Zhongyou 821. Chin. Oilseed Crops 3:1-5.

12. Hoffman, D. D., Diers, B. W., Hartman, G. L., Nickell, C. D., Nelson, R. L., Pedersen, W. L., Cober, E. R., Graef, G. L., Steadman, J. R. Grau, C. R., Nelson, B. D., del Rio, L. E., Helms, T., Anderson, T., Poysa, V., Rajcan, I., and Stienstra, W. C. 2002. Selected soybean plant introductions with partial resistance to Sclerotinia sclerotiorum. Plant Dis. 86:971980.

13. Kim, H. S., and Diers, B. W. 2000. Inheritance of partial resistance to Sclerotinia stem rot in soybean. Crop Sci. 40:55-61.

14. Kim, H. S., Hartman, G. L., Manandhar, J. B. Graef, G. L., Steadman, J. R., and Diers, B. W. 2000. Reaction of soybean cultivars to Sclerotinia stem rot in field, greenhouse, and laboratory evaluations. Crop Sci. 40:665-669.

15. Kim, H. S., Sneller C. H., and Diers, B. W. 1999. Evaluation of soybean cultivars for resistance to Sclerotinia stem rot in field environments. Crop Sci. 39:64-68.

16. Kolkman, J. M., and Kelly, J. D. 2000. An indirect test using oxalate to determine physiological resistance to white mold in common bean. Crop Sci. 40:281-285

17. Kolkman, J. M., and Kelly, J. D. 2002. Agronomic traits affecting resistance to white mold in common bean. Crop Sci. 42:693-699.

18. Kolkman, J. M., and Kelly, J. D. 2003. QTL conferring resistance and avoidance to white mold in common bean. Crop Sci. 43:539-548.

19. Mariani, C., Gossele, V., de Beuckeleer, M., de Block, M., Goldberg, R. B., de Greef, W., and Leemans, J. 1992. A chimeric ribonucleaseinhibitor gene restores fertility to male-sterile plants. Nature 357:384-387.

20. Maxwell, D. P., and Lumsden, R. D. 1970 Oxalic acid production by Sclerotinia scle- 
rotiorum in infected bean and in culture. Phytopathology 60:1395-1398.

21. Miklas, P. N., Johnson, W. C., Delorme, R., and Gepts, P. 2001. QTL conditioning physiological resistance and avoidance to white mold in dry bean. Crop Sci. 41:309-315.

22. Mithen, R. 1992. Leaf glucosinolate profiles and their relationship to pest and disease resistance in oilseed rape. Euphytica 63:71-83

23. Mithen, R. F., Lewis, B. G., FenwicK, G. R., and Heaney, R. K. 1987. Resistance of Brassica species to Leptosphaeria maculans. Trans. Br. Mycol. Soc. 88:525-531.

24. Nelson, B. D., Helms, T. C., and Olson, M. A. 1991. Comparison of laboratory and field evaluation of resistance in soybean to Sclerotinia sclerotiorum. Plant Dis. 75:662-665.

25. Oil Crops Situation and Outlook Yearbook. Market and Trade Economics Division, Economic Research Service, U. S. Department of Agriculture, October 2002, OCS-2002.

26. Orellana, R. G. 1975. Photoperiod influence on the susceptibility of sunflower to Sclerotinia stalk rot. Phytopathology 65:12931298.

27. Pratt, R. G., and Rowe, D. E. 1995. Compara- tive pathogenicity of isolates of Sclerotinia trifoliorum and S. sclerotiorum on alfalfa cultivars. Plant Dis. 79:474-477.

28. Purdy L. H. 1979. Sclerotinia sclerotiorum: history, diseases and symptomatology, host range, geographic distribution and impact. Phytopathology 69:887-890.

29. Schwartz, H. F., and Steadman, J. R. 1978. Factors affecting sclerotiorum populations of, and apothecium production by, Sclerotinia sclerotiorum. Phytopathology 68:383-388.

30. Shah, D. A., and Madden, L. V. 2004. Nonparametric analysis of ordinal data in designed factorial experiments. Phytopathology 94:3343.

31. Sun, C. 1995. Comparison of methods for evaluating oilseed cultivars for resistance to Sclerotinia sclerotiorum in Brassica napus L. Acta Agric. Shanghai 11:17-22.

32. Teo, B. K., Morrall, R. A. A., and Verna, P. R. 1989. Influence of soil moisture, seeding date, and canola cultivars (Tobin and Westar) on the germination and rotting of sclerotia of Sclerotinia sclerotiorum. Can. J. Plant Pathol. 11:393-399.

33. Thompson, C., Dunwell, J. M., Johnstone, C.
E., Lay, V., Ray, J., Schmitt, M., Watson, H., and Nesbet, G. 1995. Degradation of oxalic acid by transgenic oilseed rape plants expressing oxalate oxidase. Euphytica 85:169-172.

34. Turkington, T. K., and Morrall, R. A. A. 1993. Use of petal infestation to forecast Sclerotinia stem rot of canola: the influence of inoculum variation over the flowering period and canopy density. Phytopathology 83:682-689.

35. Vuong, T. D., Hoffman, D. D., Diers, B. W. Miller, J. F., Steadman, J. R., and Hartman, G L. 2004. Evaluation of soybean, dry bean, an sunflower for resistance to Sclerotinia scle rotiorum. Crop Sci. 44:777-783.

36. Wegulo, S. N., Yang, X. B., and Martinson, C. A. 1998. Soybean cultivar responses to Sclerotinia sclerotiorum in field and controlled environment studies. Plant Dis. 82:1264-1270.

37. Zhao, J., and Meng, J. 2003. Genetic analysis of loci associated with partial resistance to Sclerotinia sclerotiorum in oilseed Brassica napus. Theor. Appl. Genet. 106:759-764.

38. Zhou, L. C., Yu, Q., Liu, S. Y., and Zhou, B. W. 1994. Evaluation of Sclerotinia resistance in rapeseed. Chin. Oilseed Crops (Suppl.) 4:69-72. 